\title{
The System Design of Vision-Based Intelligent Robots
}

\author{
Yuanqin Zhang, Xiangjun Zou*, Shaofeng Luo, Lufeng Luo and Weiming Situ \\ College of Engineering, South China Agricultural University, Guangzhou, China \\ ${ }^{*}$ Corresponding author
}

\begin{abstract}
Equipping with a vision system for robot is an important tendency for the development of intelligent robots. To explore the applications problems of visual robot in wood processing, we have designed vision-based wood stacking robot system, the system has combined visual system and industrial robots, using vision to recognize and locate targets, and then robots can achieve wood stacking tasks automatically. In experiment, we've built a visual platform and simulated wood processing workspace, then the system uses robot to complete wood identification, classification and stacking work. The experiment shows that the visual system can drive robot to complete the work stably and accurately.
\end{abstract}

Keywords-visual system; robot; wood; location; stacking

\section{INTRODUCTION}

In recent years, the forest industry has shown the extent of investment funds, the growth mode of commercial and the improvement of industry concentration. China needs the forest and its products in the future with the fast economic development ${ }^{[1]}$. According to the development prospects of China's wood industry, it is necessary to improve the production technology and equipment of woodworking in the face of current insufficient workers and worse situation of the production environment. Traditional industrial robot can only execute scheduled commands in a strictly defined structured environment, lacking of environmental awareness and adaptability, which greatly limits the application of robots ${ }^{[2]}$. Vision systems is tending to use software and hardware modules to complete image processing and calculation functions, improving speed and accuracy ${ }^{[3]}$.Equipped with Visual systems for industrial robots, using the robot visual control, you do not need prior to teaching or off-line programming to the trajectory of the industrial robot ,you can save a lot of programming time ,improve production efficiency and quality ${ }^{[4]}$. In the field of research in robotic visual, Visual localization technology is a branch of the most active filed and an important symbol of development of robot technology ${ }^{[5]}$.Visual systems used in industrial robots, which makes the robot have the function of the human eye, is the focus of the robot research ${ }^{[6]}$. In view of this, this article has explored how to apply visual robot to wood processing, and combined the vision system and the robot by image processing technology, which makes the robot equipped with visual systems to replace human hard and tedious work, so as to improve production efficiency and quality.

\section{SYSTEM FRAMEWORK}

The system of vision-based wood stacking robots has been composed of visual systems and body structure. The system architecture has been shown in Figure 1. The vision system has been responsible for the identification and location of the wood, the image recognition algorithm has been used to find the position of the wood in the image, and the spatial coordinates of the wood have been obtained through the 3D visual analysis. Then, the space coordinates of the wood have been sent to the robot body control system by the vision system. The robot control system has been used to grab and place the wood according to the wood stacking path, ultimatly terminating the wood stacking operations.

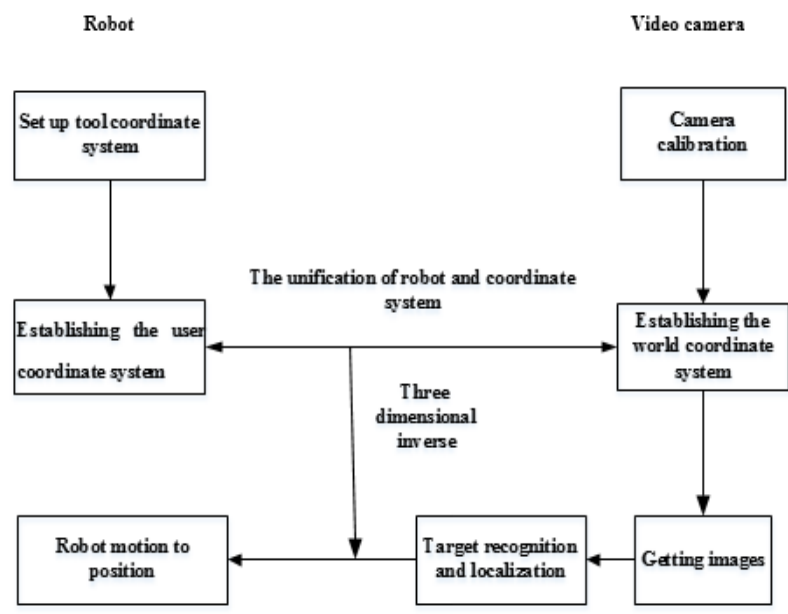

FIGURE I. SYSTEM FRAME DIAGRAM

\section{EQUIPPED WITH THE END EFFECTOR AND VISUAL MODULE}

\section{A. Assemble End-Effector}

From the point of the shape, the industrial robot is similar to the human arm, it is composed of a series of rigid Rod through a series of alternating flexible joint connected to open chain. It's joint is equivalent to one shoulder, elbow and wrist joints. The front of the operating arm with the end effector or the corresponding tool, called gripper, gripper is made up of two or more fingers, fingers can be used to "open" and "close", realize the grab action and subtle operation ${ }^{[7]}$. In this paper using a six-axis industrial robot, when the sixth axis of the 
robot itself is equipped with tool, for the convenience to take point, it is necessary to set the corresponding tool frame, moving the robot motion control points from J6 axis of the flange center to the tool center point.

There are three kinds of methods of setting the tool coordinate system, which are direct input method, the three point method and the five point method. The design of the system has adopted the three point method to set the tool coordinate system. Selecting a space-fixed point as the reference point, move the tool center point respectively in three directions (the three directions best difference of 90 degrees and is not in a plane. )near the point of reference, the system automatically calculate the tool center point coordinates value relative to the Center flange . In the process of the experiment design, the center of the end effector is installed on a tip as the auxiliary tool center point, when the tool coordinate system setting successfully, need to remove the auxiliary tip, at the same time to modify the tool coordinate system, using three coordinate directions $(\mathrm{X}, \mathrm{Y}, \mathrm{Z})$ coordinate value coefficient according to the same proportion (the ratio of auxiliary tip length and tool coordinate system $\mathrm{Z}$ coordinate value) decreased, thus, the new tool coordinate system will be set up.

\section{B. Camera Calibration Method}

The camera calibration is designed to calculate the 3D geometric information of the object from the acquired image, and it is the key step to reconstruct and recognize the object ${ }^{[8]}$. The accuracy of the calibration results and the calibration method will determine the accuracy of subsequent correction, removal of distortion, and reconstruction of 3D objects ${ }^{[9]}$. This paper has adopted the zhangzhengyou calibration method to camera calibration, this method can have accurately calibrated only needing a plurality of checkerboard images.it is by the image acquisition board corner coordinates, and seting up the world coordinate system on the checkerboard, and according to several groups of world coordinates and image coordinates to solve the camera matrix method of internal and external parameters $^{[10][11]}$.

\section{Establishing the World Coordinate System}

World coordinate system is established in order to get transformation of the world coordinate system and camera coordinate system, namely the rotation matrix and translation matrix between the world coordinate system and the camera coordinate system. World coordinate system is arbitrary, and it represents the absolute coordinates of the object, so put a calibration board, which is determined by the calibration board corner the world coordinate system. Using the camera to get the calibration board image to obtain the rotation matrix and translation matrix between the world coordinate system and the camera coordinate system.

\section{Establishing the User Coordinate System}

In the allowable range of the robot motion, according to the specific needs of users needs to establish a right angle coordinate system which is suitable for the plane on a new plane that is the user coordinate system. $\mathrm{n}$ the user coordinate system, the robot moves along the axis of the specified user coordinate system. There are two ways to set the user coordinate system: direct input method and the three point method. In this experiment, the three point method is used to set the user coordinate system. In the original plane work bench, move the robot to calibration board of the angular position in the upper left corne and record the origin of the user coordinate system, then move the robot along the $+X$ direction of the desired user to at least $250 \mathrm{~mm}$ and record the $+\mathrm{X}$ direction point, last move the robot along the $+\mathrm{Y}$ direction of the desired user(perpendicular to $+X$ direction) to at least $250 \mathrm{~mm}$ and record $+Y$ direction point. After you get the three points, the system automatically calculates the user relative to the robot base coordinate system of six-dimensional vectors $[\mathrm{X}, \mathrm{Y}, \mathrm{Z}, \mathrm{A}, \mathrm{B}, \mathrm{C}]$ coordinate values.

\section{WOOD POSITIONED}

\section{A. Gauss Filter}

There will be a lot of random noise in the original image, which will affect the image processing. In this experiment, Gauss filter is used to smooth the image. Gauss filter is a kind of linear smoothing filter, which is based on the shape of the Gauss function to select the weights, limitting the distribution of noise is very effective ${ }^{[12]}$.For image processing, two dimensional zero -mean discrete Gauss function is used as the smoother, and the two-dimensional Gauss function is as follows:

$$
G_{0}(x, y)=A e \frac{-\left(x-u_{x}\right)^{2}}{2 \sigma_{x}{ }^{2}}+\frac{-\left(y-u_{y}\right)^{2}}{2 \sigma_{y}{ }^{2}}
$$

Among them, the Sigma of Gaussian distribution parameters determines the width of the Gaussian function and the degree of smoothing ${ }^{[13]}$.

\section{B. Contour Extraction}

First of all, the edge detection is to identify the pixels in the image with obvious changes in brightness, steps is generally filtered enhanced and detected ${ }^{[14]}$.This experiment adopts Canny edge detection operator, then the extraction alone detected edges are stored in a two-dimensional container to get multiple target the outermost contour, the contour extraction results as shown in Figure 2.

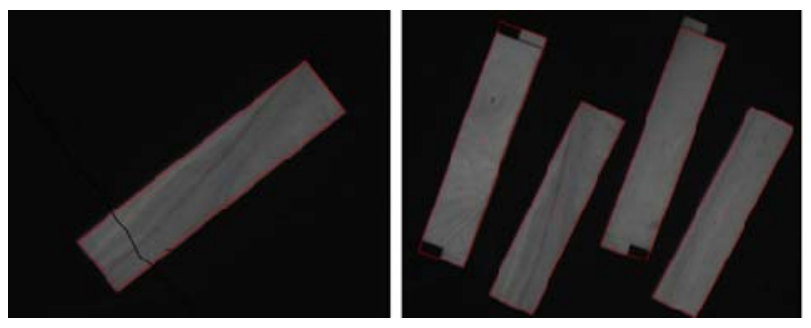

FIGURE II. CONTOUR EXTRACTION RESULTS

\section{Centroid Calculation}

After the outer contour extraction is completed, the pixels on the contour are stored in the form of point vector. Find all 
the pixels in the vector coordinates value $(\mathrm{X}, \mathrm{Y})$ of the firstorder moment (expected) to calculate the centroid coordinates of wood. Then the robot can locate the centroid position with picking up the wood.

\section{WOOD STACKING}

\section{A. The Identification of Wood Rotation Angle}

Woods are placed on the table at random. In order to stack the wood neatly, it is necessary to identify the rotation angle of the wood relative to the horizontal plane of the table. To identify the rotation angle of the wood, it is feasible to find out the smallest external rectangle of the wood contour, and then calculate the angle between the long edge of the rectangle and the horizontal plane of the worktable.

\section{B. Planning Path}

Move the robot according to the following schematic shown in the left of Figure 3 to reach in turn the teaching point $(1,2,3,4)$ and recorded. When the wood is placed on the worktable, the robot recognizes the wood and absorbs the wood. Based on the experimental design of wood stacked form as shown in the right of Figure 3 , the robot path planning of wood stacking task includes the four specific actions, in turn up the stack of layers.

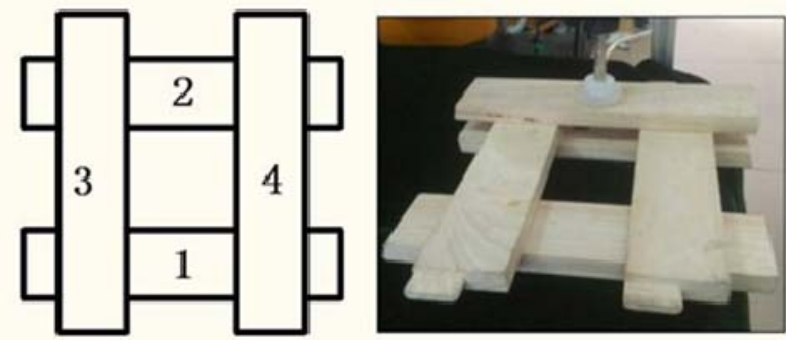

FIGURE III. SCHEMATIC DIAGRAM OF WOOD STACKING

Specific actions of the robot:

Action 1: the robot absorbs the wood to reach a certain height above the teaching point 1 and simultaneously rotates the wood to make the long edge of the smallest external rectangle of the wood contour parallel to the horizontal plane of the worktable plane. Then, the robot absorbs the wood and releases the wood after dropping a certain height. Finally, the robot will return to the origin (reset) to continue the next action 2.

Action 2:the robot absorbs the wood to reach a certain height above the teaching point 2 (appropriate distance from the teaching point 1 ) and simultaneously rotates the wood to make the long edge of the smallest external rectangle of the wood contour parallel to the horizontal plane of the worktable plane. The smallest outer contour rectangle of the wood. Then, the robot absorbs the wood and releases the wood after dropping a certain height. Finally, the robot will return to the origin (reset) to continue the next action 3 .

Action 3:firstly the robot absorbs the wood to reach a certain height (the need to consider the thickness of wood) above the eaching point 3 and simultaneously rotates the wood to make the long edge of the smallest external rectangle of the wood contour parallel to the horizontal plane of the worktable plane. Then, the robot needs to rotate the wood to 90 degrees and then release it after dropping a certain height. Finally, the robot will return to the origin (reset) to continue the next action 4.

Action 4:the robot absorbs the wood to reach a certain height (the need to consider the thickness of wood) above the teaching point 2 (appropriate distance from the teaching point 3) and simultaneously rotates the wood to make the long edge of the smallest external rectangle of the wood contour parallel to the horizontal plane of the worktable plane. The smallest outer contour rectangle of the wood. Then, the robot needs to rotate the wood to 90 degrees and then release it after dropping a certain height. Finally, the robot will return to the origin (reset) to continue the next action.

\section{PROBlEMS AND ANALYSIS OF EXPERIMENT}

In the course of the experiment, some of the end effector of the robot has no tip, and can not use the three point method to get the tool coordinate system. In order to solve this problem, we choose the end-effector equipped with an auxiliary tip. As shown in Figure 4, when the tool coordinate system setting up successfully, need to remove the auxiliary tip and adjust the coordinate values of the tool coordinate system. The solution will bring the system error, while the error did not cause a significant impact on the experiment, but if you want to apply this system to other objects, you need to take into account the existence of the error, or by other means to establish the tool coordinate system, or modify new tools coordinate system.

In addition, taking into account the actual environment of the wood processing site, the shadow of the wood itself have an impact on the image processing, so the experimental work platform should choose a dark background to eliminate the shadow effect.
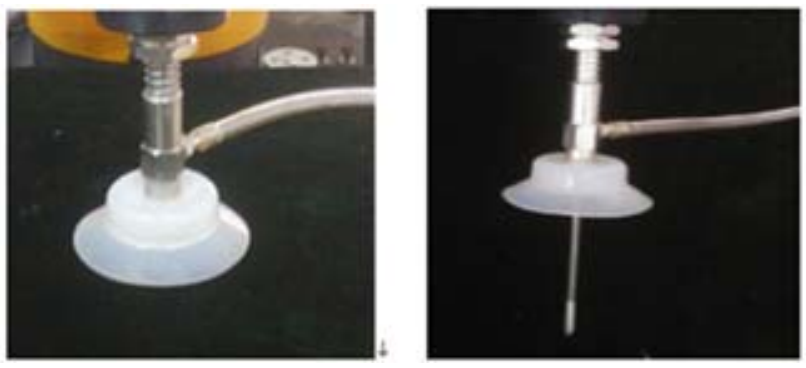

FIGURE IV. END EFFECTOR

\section{SUMMARY}

This paper has been designed to simulate the static processing system of wood processing site. the use of visual modules located the target workpiece, and then used the sixaxis robot to obtain the target in orde to complete the task stack. In this system, the vision system has been combined with the industrial robot by image processing technology and the realization of the intelligent robot in the processing of wood has been explored, which has been practical in engineering. At the same time, the test has set up a platform 
for the further study of visual recognition algorithm, as shown in Figure 5.

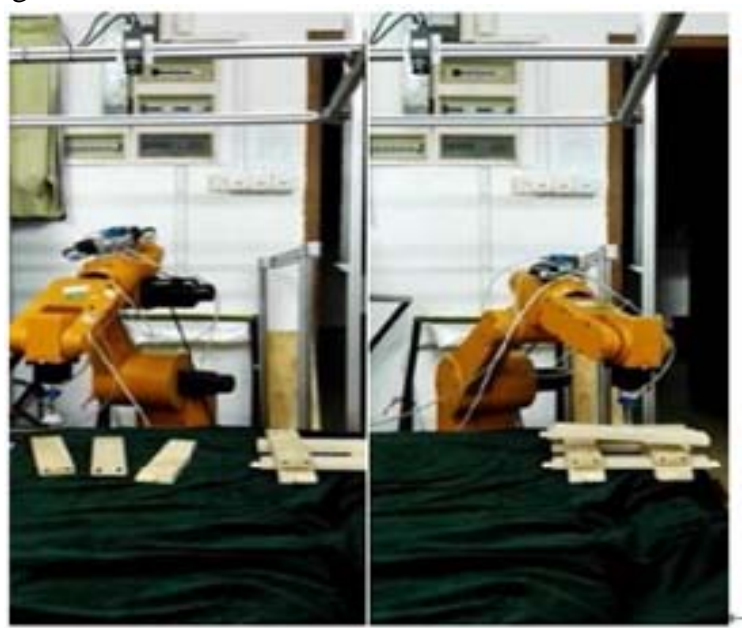

FIGURE V. EXPERIMENTAL PLATFORM

\section{ACKNOWLEDGEMENTS}

This work was financially supported by the National Natural Science Foundation of China (No.31571568), Supported by Science and Technology Planning Project of Guangdong Province.(No.2014A010104011), science and technology project of Guangzhou city (No.201510010140), science and technology project of Huizhou city (No.2014B040008006). Corresponding Author: Xiangjun ZOU.

\section{REFERENCES}

[1] China wood processing market status and development trend analysis report 2016. China Industry Research Network,2016.

[2] L.Wanhui,L.Lin.Stereo Vision for Tracking and Location System Working on Industrial Robot [J].Robot technology,2009,25(32):242.

[3] C.Lihong.Research and Design on Robot Vision System [D] Zhejiang:Zhejiang Unversity (Supported by National Laboratory of Industrial control Technology), 2003.

[4] D.Wenhui.Research on the technology of industrial robot grasping based on machine vision [D].Wuhan: Huazhong University of Science and Technology,2011.

[5] Z.Dongwei. The application of vision positioning in food detection and welding [D].Jilin: Jilin University,2015.

[6] L.Zhenyu,L.Zhongsheng,Z.Xue.Z.Fengshan. Research of sorting technology based on industrial robot of machine vision [J]. Manufacturing automation,2013,35(09):25.

[7] X.Youlun. Robot technology foundation [M].Wuhan: Publishing House of Huazhong University of Science and Technology,1995.

[8] X.Li,Y.Sheng.The Research of Camera Calibration Algorithm [J].Science \& technology view,2011(25):22.

[9] L.Yuting.Research on Robot Calibration Base on Vision [D].Guangzhou: South China University of Technology,2013.

[10] W.Zheng,H.Zhixiong. Linear and Nonlinear Solution of Internal Parameters Based on Zhang Zhengyou Calibration Algorithm [J]. Journal of Zhengzhou Normal Education, 2013(02):63-66.

[11] Zhang ZY. Flexible camera calibration by viewing a plane from unknown orientations ,International Conference on Computer Vision( ICCV’99)[C]. Kerkyra : IEEE Xplore Digital Library,1999.

[12] W.Yaogui. Analysis of image Gauss smoothing filter [J].Shandong: Computer and information technology,2008(08):79-80.
[13] M. Xingyun, L. Xuefei, W. Bihui. Introduction to OpenCV3 programming[M]. Beijing: Publishing House of Electronics Industry, 2014.

[14] Z.Liang.The Application of Machine Vision IN Grasping Technology of Industrial Robot[D].Shenyang:University of Chinese Academy of Sciences (Shenyang Institute of Computing Technology),2016. 